\title{
Comparison of weight estimation methods and evaluation of usability of Broselow Luten tape in Turkish children
}

\author{
Ayşe Gültekingil-Keser, Özlem Tekşam \\ Division of Pediatric Emergency Medicine, Department of Pediatrics, Hacettepe University Faculty of Medicine, Ankara, \\ Turkey.Email: oteksam@yahoo.com \\ Received: 16th February 2016, Revised: 6th December 2016, Accepted: 5th June 2017
}

\begin{abstract}
SUMMARY: Gültekingil-Keser A, Tekşam Ö. Comparison of weight estimation methods and evaluation of usability of Broselow Luten tape in Turkish children. Turk J Pediatr 2017; 59: 150-154.

It is controversial which method is more reliable for weight estimation of a child in an emergent situation. The aim of this study is to compare different methods and to review the applicability of Broselow Luten tape for Turkish children. This study was performed prospectively in patients presenting to pediatric emergency department. Weight estimation of patients were performed by parent, physician, nurse and Broselow Luten tape. Finally actual weight and height of the patient were measured. A total of 438 patients were eligible for the study. All estimates have a reliable correlation with actual weight. However, parent estimation is better followed by Broselow Luten tape. Correlation decreases as the weight of the child increases. When emergent care of an acutely ill child is necessary, parental estimation of the weight can be used. If it is unattainable, Broselow Luten tape can be reliably used instead of other methods especially in patients with lower weight.
\end{abstract}

Key words: Broselow Luten tape, children, pediatric emergency, weight estimation.

Pediatric emergency physicians tend to exactly know the weight of the patients when dealing with an emergent situation in order to assess and treat the patient properly. However actually weighing the patient might delay the urgent treatment, therefore many different weight estimation methods were studied ${ }^{1-4}$. It is important to use the method which is both quick and precise in order to use correct medication dose and correct equipment without wasting time ${ }^{5}$.

Broselow Luten pediatric emergency tape uses length in order to estimate weight. It has color coded zones that provide doses of drugs, intravenous fluid volumes and size of equipment calculated for the estimated weight ${ }^{2,6}$. Ease of use and relatively high accuracy of estimation made Broselow Luten tape widely used and even supported by AHA guidelines ${ }^{1,7,8}$. However, debate still continues about its validity in different age groups, different weight groups and different populations in different countries ${ }^{9-13}$.

The aim of this study is to compare different weight estimation methods including Broselow Luten pediatric emergency tape, and estimations of parents, physician and triage nurse with the actual weight of the child to understand which method is more reliable and to figure the applicability of Broselow Luten tape in Turkish children.

\section{Material and Methods}

The study is prospectively conducted in Hacettepe University Children's Hospital Pediatric Emergency Unit between January 1st 2013 and July 1st 2013. All patients presented to pediatric emergency department between 10:00 am and 12:00 pm were included in the study. Age, gender and medical condition of the patient were recorded. Weight of the patient was estimated by physician at triage (without using any formula), triage nurse and parent of the patient blindedly. Afterwards length of the patient was measured with Broselow Luten tape on supine position on examination bed and weight equivalent of the length is noted. Finally, actual length in $\mathrm{cm}$ and weight in $\mathrm{kg}$ 
were measured and recorded. Weight and height were measured on upright position if patient can stand on his own, if not measurements were done in supine position. For immobile patients, parents hold the patient on measurement, then weight of the parent was subtracted from the measured weight. Weight percentiles for age were calculated for each patient and noted.

As Broselow Luten tape is $146 \mathrm{~cm}$ and it is designed for children up to 12 years old and $36 \mathrm{~kg}$, the patients older than 12 years old, taller than $146 \mathrm{~cm}$ or heavier than $36 \mathrm{~kg}$ were excluded from the study. Patients with weight percentiles less than $3^{\text {rd }}$ percentile or more than $97^{\text {th }}$ percentile were excluded in order to analyze its appropriateness for children with average weight and height. Repeated recordings of the same patients were excluded. Our research was approved by the ethics commmitte of the university and written or verbal consent was obtained from the parents of all participants.

Statistical analysis was performed using SPSS 15.0. Cronbach's alfa coefficient for reliability, intraclass correlation coefficient (ICC) and $95 \%$ coefficient interval (CI) was used to measure overall difference between actual weight and parental, physician, nurse and Broselow Luten estimate and difference in each weight category. Bland-Altman plot was used to analyze agreement in each estimate category with actual weight.

\section{Results}

A total of 650 patients were enrolled in the study; 152 patients were excluded because they were older than 12 years. Five patients were excluded because they were taller than $146 \mathrm{~cm}$ and 13 patients were excluded because they were heavier than 36 kilograms. Fourty-two patients were excluded because weight of these patients were either less than 3rd percentile or more than 97th percentile for their age. Remaining 438 patients were included in the final analysis. Mean age of the patients was 4.27 years (1 month-12 years). One hundred ninty of the patients were girls $(43.4 \%)$ and 208 were boys $(56.6 \%)$. Mean weight of the patients was $16.98 \pm 8.81 \mathrm{~kg}$, mean height of the patients was $99.29 \pm 26.67 \mathrm{~cm}$.

All estimation methods have a reliable correlation with actual weight of the patient. Among different estimation methods, parental estimation had the highest correlation with actual estimation (ICC: 0.99; 95\% CI: 0.980.99) followed by Broselow Luten tape (ICC: 0.97; 95\% CI: 0.96-0.97). Nurse estimation was worse than parental estimation and Broselow Luten tape (ICC:0.96; 95\% CI: 0.95-0.96). Physician estimation was the worst of all four estimates (ICC: 0.94; 95\% CI: 0.93-0.95).

Bland-Altman plots show that Broselow Luten tape estimations tends to underestimate children especially when weight of children increases (Fig. 1). On the contrary estimations of nurses tend to overestimate when weight of children increases (Fig. 2). Physician estimations perform better in lower weight ranges but they tend to both over and underestimate when weight of children increases (Fig. 3). Parental estimates also perform better in low weight and its performance decreases as the weight increase however not as much as other estimates (Fig. 4).

When patients were classified according to weight percentiles, all estimates are reliable in each percentile group. Parental estimation had the highest correlation followed by Broselow Luten tape and physician estimation had the lowest correlation within each percentile group.

When patients were classified according to weight, all estimation methods perform better if patients weigh less than $10 \mathrm{~kg}$. Reliability decreases as patients' weight increases except for parental estimation which performed better in $>20 \mathrm{~kg}$ group than $10-20 \mathrm{~kg}$ group.

\section{Discussion}

Accurate and rapid weight estimation of a critically ill child in a pediatric emergency department is essential to prevent delay of treatment and dosing errors ${ }^{5,14}$. However it is difficult to decide the reliable method for weight estimation, some methods could give accurate results but they could be time consuming to calculate in an emergent situation 1,13,15. Other methods may be subject to error they can be easily effected by assessment of different individuals ${ }^{10}$.

Broselow Luten emergency tape is uncomplicated, easy to use, objective method for estimation of weight of children and provides the calculated doses of different medications and equipment which is very efficaceous in 


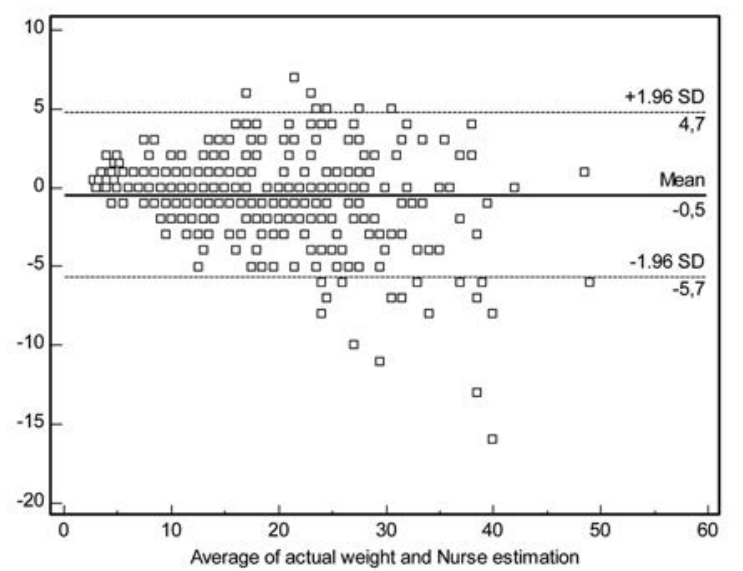

Fig. 2. Bland-Altman analysis of nurse estimate and actual weight

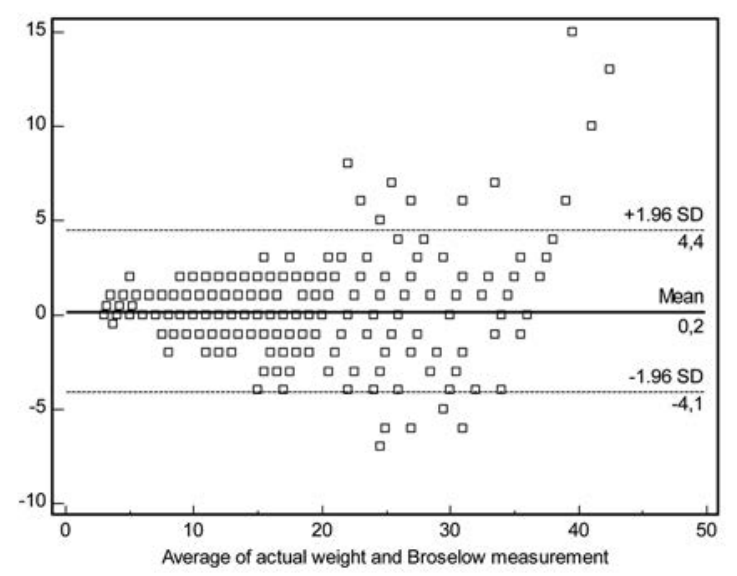

Fig 1. Bland Altman analysis of Broselow Tape

an emergent situation ${ }^{16,17}$. However there are still questions about accuracy of the tape ${ }^{9,11}$. Nieman et al. ${ }^{9}$ found that accuracy of the tape decreases with increasing age. Knight et al. ${ }^{11}$ noted that half of the children fell outside the predicted weight range and emphasized that Broselow Luten tape underestimates the weight of children. Sinha et al. ${ }^{18}$ reported that trauma stretcher integrated weighting scale gave better results than Broselow Luten tape. Age based formulas give comparable results with Broselow Luten tape ${ }^{19,20}$.

Reliability of Broselow Luten tape differs betweeen different populations and its accuracy has never been evaluated in Turkish children $12,13,21-24$. Our results showed that Broselow Luten emergency tape can be reliably used in Turkish children with average weight and height. When it was compared with other estimations, parental estimation performed

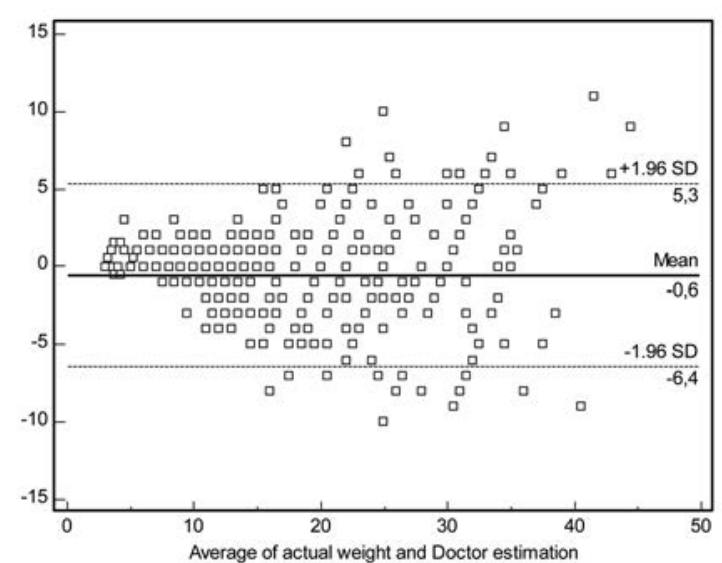

Fig. 3. Bland Altman analysis of physician estimate and actual weight

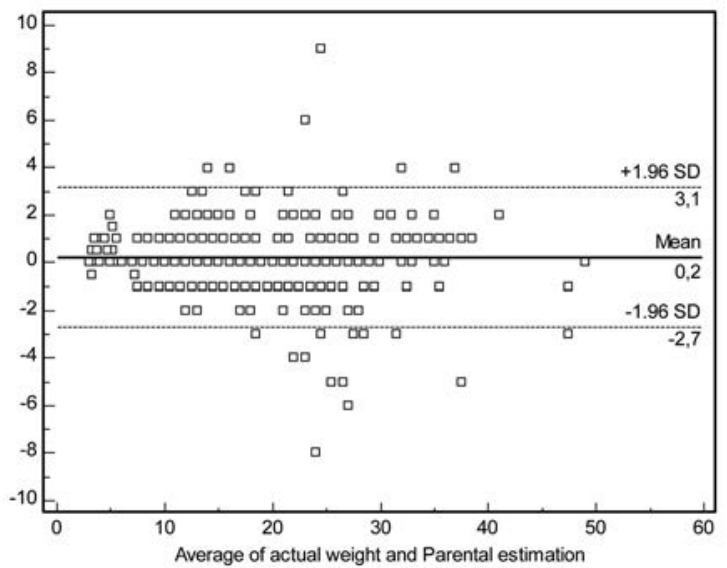

Fig. 4. Bland-Altman analysis of parental estimate and actual weight

better than Broselow Luten tape in all weight categories. Krieser et al. ${ }^{25}$ also previosuly reported that parental estimation of the weight was more accurate than Broselow Luten tape. However, Broselow Luten tape performed better than physician and nurse estimates in all weight categories, consistent with the study of Rosenberg et al. ${ }^{8}$ in which physician estimations was worse than Broselow Luten tape except in obese patients, yet obese patients were not included in our study group.

When weight categories were considered, there was no significant difference in reliability of Broselow Luten tape in different weight percentiles for age. In contrast, reliability declines when weight of the patient increases in concordance with other studies $1,9,23,26$. However reliability of other estimation methods decreases as weight of the patient increases as well it does not bring an additional disadvantage 


\section{for Broselow Luten tape.}

There are also other methods for accurate weight estimation that can be used in the emergency department. Age based methods or midarm circumference methods can result in fairly good estimation of weight especially in specific subgroups, even out performing Broselow Luten tape, however they could be time consuming in emergent situations ${ }^{26,27}$. There are newer tapes such as MERCY tape and PAWPER tape that were shown to be superior to Broselow Luten tape in weight estimation in few studies in literature. Therefore, future studies could be done to compare them with Broselow Luten tape for the Turkish population as well28,29.

Limitations of our study were that we excluded the underweight and overweight children in order to show the applicability of Broselow Luten tape on average Turkish children. However, this study would be planned on patients with underweight and overweight children. Also, we only compared Broselow Luten tape with parental and medical staff estimations but Broselow Luten tape could be compared with other estimation methods and age based formulas as well. In addition to that, results would be more reliable if more than one nurse and physician estimation could have been used and interrater reliability was also assessed, however it would be time consuming for patients at triage. Therefore, we did not have chance to perform this assessment.

This is the first prospective study to investigate the usability of Broselow Luten tape in Turkish children. Parental estimation is the best of all estimation methods; Broselow Luten tape is the second best therefore Broselow Luten tape can be used for weight estimation if parental estimation is unattainable. Broselow Luten tape can be reliably used on Turkish children in an emergency medical situation.

\section{REFERENCES}

1. So TY, Farrington E, Absher RK. Evaluation of the accuracy of different methods used to estimate weights in the pediatric population. Pediatrics 2009; 123 : e1045-1051.

2. Pinchevsky LE, Pesaturo KA, Smith BS, Hartman CA. Pilot comparison of three cardiopulmonary resuscitation medication dosing strategies in overweight children. J Pediatr Pharmacol Ther 2010; 15: 282-289.
3. Casey J, Borland M. Best guess method: a further external validation study and comparison with other methods. Emerg Med Australas 2010; 22: 68-74.

4. Young TP, Chen BG, Kim TY, Thorp AW, Brown L. Finger counting: an alternative method for estimating pediatric weights. Am J Emerg Med 2014; 32: 243-247.

5. Hoyle JD, Davis AT, Putman KK, Trytko JA, Fales WD. Medication dosing errors in pediatric patients treated by emergency medical services. Prehosp Emerg Care 2012; 16: $59-66$.

6. Deboer S, Seaver M, Broselow J. Color coding to reduce errors. Am J Nurs 2005; 105: 68-71.

7. Kleinman ME, Chameides L, Schexnayder SM, et al. Part 14: Pediatric Advanced Life Support: 2010 American Heart Association Guidelines for Cardiopulmonary Resuscitation and Emergency Cardiovascular Care. Circulation 2010; 122 (Supply 3): S876-S908.

8. Rosenberg M, Greenberger S, Rawal A, LatimerPierson J, Thundiyil J. Comparison of Broselow tape measurements versus physician estimations of pediatric weights. Am J Emerg Med 2011; 29: 482-8.

9. Nieman CT, Manacci CF, Super DM, Mancuso C, Fallon WF Jr. Use of the Broselow tape may result in the underresuscitation of children. Acad Emerg Med 2006; 13: 1011-1019.

10. DuBois D, Baldwin S, King WD. Accuracy of weight estimation methods for children. Pediatr Emerg Care 2007; 23: 227-230.

11. Knight JC, Nazim M, Riggs D, et al. Is the Broselow tape a reliable indicator for use in all pediatric trauma patients? A look at a rural trauma center. Pediatr Emerg Care 2011; 27: 479-482.

12. Ramarajan N, Krishnamoorthi R, Strehlow M, Quinn J, Mahadevan SV. Internationalizing the Broselow tape: how reliable is weight estimation in Indian children. Acad Emerg Med 2008; 15: 431-436.

13. Theron L, Adams A, Jansen K, Robinson E. Emergency weight estimation in Pacific Island and Maori children who are large-for-age. Emerg Med Australas 2005; 17: 238-243.

14. Kaji AH, Gausche-Hill M, Conrad H, et al. Emergency medical services system changes reduce pediatric epinephrine dosing errors in the prehospital setting. Pediatrics 2006; 118: 1493-1500.

15. Leffler S, Hayes M. Analysis of parental estimates of children's weights in the ED. Ann Emerg Med 1997; 30: $167-170$.

16. Ogden CL, Carroll MD, Kit BK, Flegal KM. Prevalence of childhood and adult obesity in the United States, 2011-2012. JAMA 2014; 311: 806-814.

17. Agarwal S, Swanson S, Murphy A, Yaeger K, Sharek P, Halamek LP. Comparing the utility of a standard pediatric resuscitation cart with a pediatric resuscitation cart based on the Broselow tape: a randomized, controlled, crossover trial involving simulated resuscitation scenarios. Pediatrics 2005; 116: e326-333. 
18. Sinha M, Lezine MW, Frechette A, Foster KN. Weighing the pediatric patient during trauma resuscitation and its concordance with estimated weight using Broselow Luten Emergency Tape. Pediatr Emerg Care 2012; 28: 544-547.

19. Hofer CK, Ganter M, Tucci M, Klaghofer R, Zollinger A. How reliable is length-based determination of body weight and tracheal tube size in the paediatric age group? The Broselow tape reconsidered. Br J Anaesth 2002; 88: 283-285.

20. Luten RC, Zaritsky A, Wears R, Broselow J. The use of the Broselow tape in pediatric resuscitation. Acad Emerg Med 2007; 14: 500-1; author reply 501-502.

21. Loo PY, Chong SL, Lek N, Bautista D, Ng KC. Evaluation of three paediatric weight estimation methods in Singapore. J Paediatr Child Health 2013; 49: E311-316.

22. Varghese A, Vasudevan VK, Lewin S, Indumathi CK, Dinakar C, Rao SD. Do the length-based (Broselow) tape, APLS, Argall and Nelson's formulae accurately estimate weight of Indian children? Indian Pediatr 2006; 43: 889-894.

23. Jang HY, Shin SD, Kwak YH. Can the Broselow tape be used to estimate weight and endotracheal tube size in Korean children?Acad Emerg Med 2007; 14: 489-491.
24. House DR, Ngetich E, Vreeman RC, Rusyniak DE. Estimating the weight of children in Kenya: do the Broselow tape and age-based formulas measure up? Ann Emerg Med 2013; 61: 1-8.

25. Krieser D, Nguyen K, Kerr D, et al. Parental weight estimation of their child's weight is more accurate than other weight estimation methods for determining children's weight in an emergency department? Emerg Med J 2007; 24: 756-759.

26. Cattermole GN, Leung MP, So HK, et al. Age-based formulae to estimate children's weight in the emergency department. Emerg Med J 2011; 28: 390-396.

27. Cattermole GN, Leung PY, Mak PS, Graham CA, Rainer TH. Mid-arm circumference can be used to estimate children's weights. Resuscitation 2010; 81: 1105-1110.

28. Abdel-Rahman SM, Paul IM, James LP, Lewandowski A. Evaluation of the Mercy TAPE: performance against the standard for pediatric weight estimation. Ann Emerg Med 2013; 62: 332-339.

29. Wells M, Coovadia A, Kramer E, Goldstein L. The PAWPER tape: A new concept tape-based device that increases the accuracy of weight estimation in children through the inclusion of a modifier based on body habitus. Resuscitation 2013; 84: 227-232. 